\title{
Non sex-related subtotal rupture of the corpus cavernosum without urethral injury: A case report and literature review
}

\author{
Zeki Bayraktar, Selami Albayrak \\ Department of Urology, Istanbul Medipol University, School of Medicine, Istanbul, Turkey.
}

\begin{abstract}
Summary Although penile fracture is a rare case, it is a well-described urologic emergency. It results from the rupture of the tunica albuginea of corpora cavernosa by blunt strain that commonly mandates immediate surgical exploration. Urethral injury may also accompany penile fracture. An ideal anamnesis and a special physical examination were determinant to achieve a correct diagnosis. It is usually diagnosed based on clinical examination, but ultrasonography can be very helpful in diagnosis. The treatment is based on the presence of associated urethral injury. Early surgery is preferable to conservative management, because it is associated with better outcomes and fewer long-term complications. The surgical repair of cavernous body can produce good results, with a favorable prognosis and minimal rate of complications. We present a penile fracture case of 34-year-old with subtotal rupture of the right corpus cavernosum without urethral injury who treated by early surgery and good results.
\end{abstract}

KEY WORDS: Penile fracture; Rupture; Diagnosis; Treatment; Surgery.

Submitted 12 April 2016; Accepted 22 May 2016

\section{INTRODUCTION}

Penile fracture is a urological emergency which is caused by the rupture of the tunica albuginea of the corpus cavernosum following a blunt trauma to the erect penis (1). Tunical rupture caused by non-physiological bending of the penile shaft can be in one or two corpus cavernosum and it may be accompanied by urethral injury. The incidence of concomitant injury of the urethra is $0-38 \%$ in literature (2).

We report the surgical repair of a penile fracture including subtotal disruption of corpus cavernosum without urethral injury. In this case, despite there was a subtotal rupture of the right corpus cavernosum, no urethral injury was observed.

\section{Case report}

A 34 -year-old man presented with rapid detumescence, acute penile pain, penile swelling and deviation to left side following a cracking sound at penile manipulation 2 hours before in January 2016. The patient was married. But he did not declare sexual intercourse. On physical examination, there was swelling and subcutaneous hematoma in the penis (on the right penis skin). The defect at the fracture site was palpable. No hematoma was in the scrotum and pubis. Penis presented left-sided deviation (Figure 1). There was no urethral discharge and also no microscopic hematuria was found in the urine analysis. Patient did not declare dysuria or any other urination problem. Penile ultrasonography showed the presence of haematoma in the proximal shaft of the penis $(30 \times 21 \mathrm{~mm})$ with a rupture of the tunica albuginea of the right cavernosal corpora $(10 \mathrm{~mm})$ and oedema of the subcutaneous tissue. Cavernosal bleeding from the albugineal tears was showed at penile color Doppler ultrasonography. The patient was a healthy man with no medications or allergies. He was taken to the operating room for emergency surgery. Under the general anesthesia, penile subcoronal circumcising incision was made and the penis degloved. Hematoma was limited by Buck fascia (Figure 2). Fascia was incised and hematoma was evacuated (Figure 3).

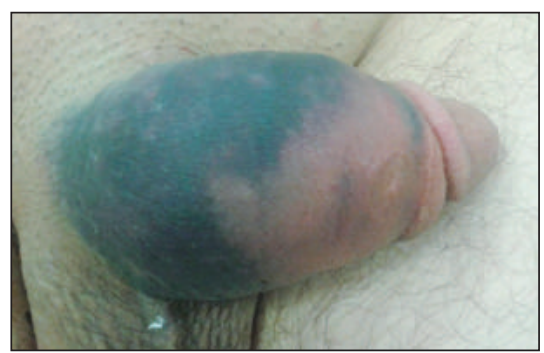

Figure 1.

Penis presenting left-sided deviation.

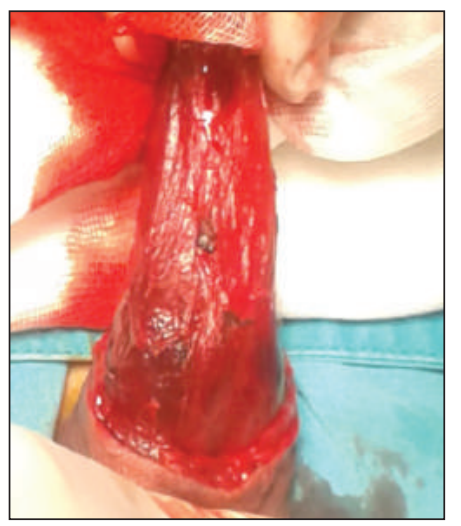

Figure 2. Hematoma limited by Buck fascia. 


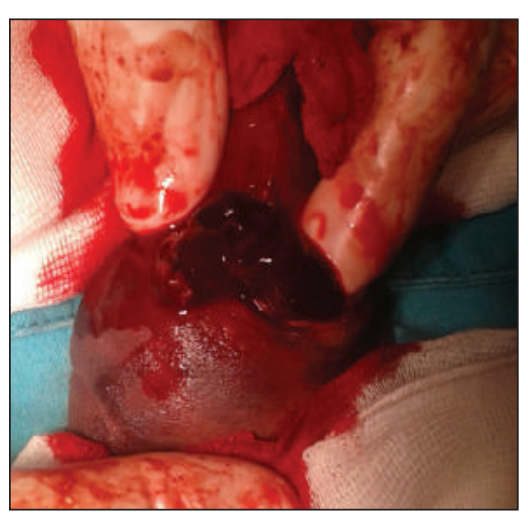

Figure 3.

Fascia incision and hematoma evacuation.

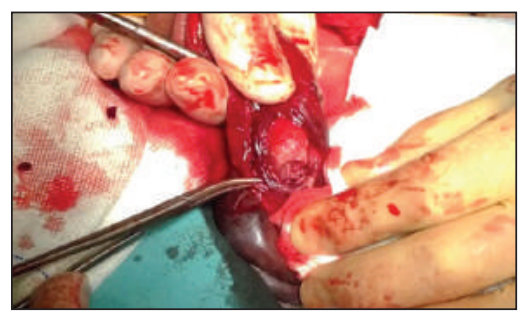

Figure 4.

Rupture almost complete of the dorsal wall of the right corpus cavernosum.

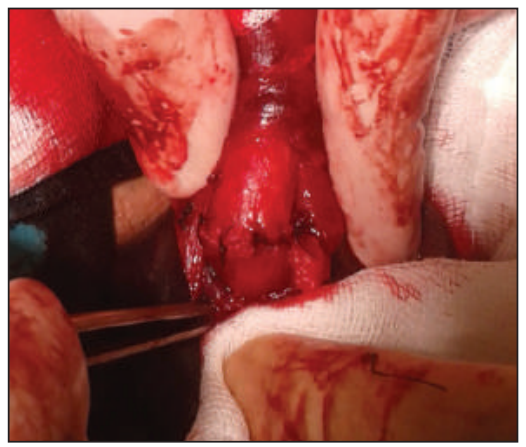

Figure 5.

Repair of the defect/rupture of the right corpus cavernosum by interrupted $3 / 0$ vicryl suture.

There was subtotal transverse tears on the right tunica albuginea/corpus cavernosum.

There were complete rupture on ventral, medial and lateral walls of the right corpus cavernosum.

Only a part of dorsal wall of the right corpus cavernosum was not ruptured (Figure 4).

\section{Correspondence}

Zeki Bayraktar, MD (Corresponding Author)

zbayraktar@medipol.edu.tr

Istanbul Medipol University, School of Medicine, Department of Urology, Medipol Sefaköy Hospital Tevfikbey Mah. Maslakçeșme Cad.No:30 Küçükçekmece, Istanbul, Turkey

Selami Albayrak, MD

salbayrak@medipol.edu.tr

Istanbul Medipol University, School of Medicine, Department of Urology, Istanbul, Turkey
A 18-French urethral catheter was passed into the bladder. Defect/rupture of the right corpus cavernosum was repaired by interrupted 3/0 vicryl sutures (Figure 5). For leakage control, artifical erection was provided by saline injected into the right corpus cavernosum. There was no leakage. Urethral catheter was removed at postoperative first day and the patient was discharged with oral antibiotic treatment.

The patient was advised to abstain from sexual intercourse for at least 4 weeks. One month after surgery, the patient was clinically evaluated for the presence of erectile dysfunction and penile deformity. Despite of the ban on sexual intercourse, the patient declared sexual intercourse at postoperative twenty-third day. There was no penile deformity in physical examination. Only a minimal induration was palpated at the fracture repair line. Patient had normal erectile and voiding functions. No problem such as pain and curvature in erection was declared by patient.

\section{Discussion and Supplementary References are posted} on www.aiua.it

\section{Conclusions}

Penile fracture is a rare condition and is generally diagnosed by clinical findings. Ultrasonography is a useful technique for confirmation of clinically suspected corpus cavernosum rupture and most often is sufficient for diagnosis (3). Some cases may also be treated as a cosmetic, emergency surgical exploration and treatment is the best guarantee of good functional and cosmetic results. Early surgery has a high success rate and low complication in penile fracture.

\section{References}

1. Eke N. Fracture of the Penis. Br J Surg. 2002; 89:555-65.

2. Kamdar C, Mooppan UM, Kim H, et al. Penile fracture: preoperative evaluation and surgical technique for optimal patient outcome. BJU Int. 2008; 102:1640-44.

3. Koifman L, Barros R, Júnior RA, et al. Penile fracture: diagnosis, treatment and outcomes of 150 patients. Urology. 2010; 76:1488-92. 